\title{
Nonoperative Treatment Compared with Plate Fixation of Displaced Midshaft Clavicular Fractures in Adults
}

\author{
AHMED HEGAZY, M.Sc.; AHMED AL-OLEMY, M.D.; MOHAMMED QUOLQELA, M.D. and \\ MOHAMMED ROMEIH, M.D.
}

The Department of Orthopedic Surgery, Faculty of Medicine, Tanta University

\begin{abstract}
Background: Fracture clavicle is one of the most common fractures, it constitutes about $4 \%$ of all fractures. $76 \%$ of clavicular fractures are fracture of the mid shaft. Clavicular fractures have classically been treated conservatively with favourable results. However, unsatisfactory results were found in recent clinical series as nonunion and malunion. The debate is still present between conservative and operative treatment of displaced fracture clavicle. This study is meant to compare the outcomes of nonoperative conservative treatment and operative treatment with plate fixation of the completely displaced midshaft clavicular fractures to clarify the merits and possible disadvantages of each method.
\end{abstract}

Aim of Study: Comparison between the outcomes of the non-operative and operative treatment of displaced mid-shaft clavicular fracture to reveal the advantages and disadvantages of each method to refine the indications of each method.

Patients and Methods: This study was performed on fourty adult patients with mid-shaft clavicular fracture, sub grouped randomly in two matched groups regarding age, sex and type of fracture. 20 patients (Group A) were treated nonoperatively by immobilization with broad arm sling and 20 patients (Group B) were treated operatively by open reduction and internal fixation (ORIF) with plate and screws. Patients were collected from orthopedic surgery department, Tanta University Hospitals, followed up at a period of 18 months and assessed using the constant shoulder score (CSS).

Results: Results were functionally satisfactory in $90 \%$ of group A patients and in $70 \%$ of group B patients according to CSS. ORIF was attended with higher complication rate, inferior overall outcome and more unsatisfied patients.

Conclusions: According to the study results, non-operative treatment by broad arm sling is the preferred line of treatment in solitary closed uncomplicated mid-shaft clavicular fractures. ORIF is recommended in cases of associated scapular neck fracture "floating shoulder".

Key Words: Fracture mid-shaft clavicle - Nonoperative VS operative treatment - Plate fixation - Displaced fracture - Comparative study.

Correspondence to: Dr. Ahmed Hegazy, The Department of Orthopedic Sugery, Faculty of Medicine, Tanta University

\section{Introduction}

CLAVICULAR fractures constitute about $4 \%$ of all fractures. They are commonly encountered as fractures of the middle third in approximately $76 \%$ of all clavicle fractures [1]. The incidence of the injury is characterized by a bimodal age distribution with peaks at the ages of younger than 40 years and older than 70 years [2]. In the elderly, clavicular fractures are primarily occurring in females, caused by a low-energy mechanism of injury such as a fall from a standing height. In the young, clavicular fractures are found predominantly in males and are secondary to a high energy direct blow sustained in sports, a motor vehicle crash, or a fall from a significant height $[1,3,4]$.

The clavicle has peculiar anatomic features, it is S-shaped with a wide medial part convex forward and a thin lateral part concave forward, both medial and lateral ends are flattened. The junction between the two cross-sectional configurations occurs in the middle third and constitutes a vulnerable area to fracture.

Clavicular fractures have classically been treated conservatively with favourable results that have been achieved specially in closed, stable, minimally displaced fractures [2]. However, in recent clinical series, unsatisfactory results were found in $4.5 \%$ up to $31 \%$ of patients after non-surgical treatment of the completely displaced midclavicular fractures as nonunion, malunion, shoulder dysfunction and cosmetic disfigurement [6-9].

Internal fixation of clavicular fractures is recommended in comminution greater than $2 \mathrm{~cm}$ of shortening, complete displacement with no cortical contact between both fragments, superior suspensory shoulder complex (floating shoulder) that 
occurs with associated fracture neck of scapula, open fractures and skin tenting with the potential for progression to open fracture [5,7]. There are different methods for internal fixation. However, open reduction and internal fixation with plate and screws is considered the gold standard method of internal fixation $[\mathbf{1 0 , 1 1 ]}$

This study is meant to compare the outcomes of nonoperative conservative treatment and operative treatment with plate fixation of the completely displaced midshaft clavicular fractures to clarify the merits and possible disadvantages of each method. In order to get a valid comparison, the patients will be grouped randomly into two matched groups regarding age, sex, type of fracture. Special attention will be paid to the clinical outcomes and complications.

\section{Classification:}

The classification system that was used in the present study was Robinson's classification in 1998 [4] as follow:

- Type 1: The fifth of the bone lying medial to a vertical line drawn upwards from the center of the first rib.

- Type 2: The intermediate three-fifths of the bone (mid-clavicular region).

- Type 3: The fifth of the bone lateral to a vertical line drawn upwards from the center of the coracoid process.

Fractures are furtherly subdivided into subgroup A (un-displaced) and subgroup B (displaced) according to the displacement of the main fragments (more or less than $100 \%$ translation).

\section{Patients and Methods}

This is a randomized, double blind, prospective and retrospective clinical study, including 40 adult patients having unilateral displaced mid-shaft closed clavicular fractures. Patients were grouped randomly into two matched groups regarding age, sex and type of fracture. Group A (non-operative group) were treated non-operatively using arm sling immobilization. Group B (operative group) were treated by open reduction and internal fixation by plate and screws. The study was conducted on patients of orthopedic surgery department, Tanta University Hospitals, from April 2017 to October 2018.

\section{Inclusion criteria:}

1- Adult patients, 18 years and older.

2- Displaced closed midshaft clavicular fracture with overlapping more than $2 \mathrm{~cm}$.
3- No medical contraindications to general anesthesia.

4- Provided informed consent.

\section{Exclusion criteria:}

1- Age below 18 years.

2- Fractures involving the proximal or distal third of the clavicle.

3- Pathological fractures.

4- Open fractures.

5- Neglected cases (fracture seen more than 14 days after injury).

6- Associated injury of neurovascular bundle.

\section{Surgical technique:}

The operation is performed under general anesthesia and the patient is placed in a semi-sitting position with a pad behind the shoulder to elevate it. The entire shoulder girdle is prepared and draped so that the upper extremity can be used to freely mobilize the lateral fragment of the fractured clavicle to aid in reduction.

The incision is centered over the fracture site. The skin and subcutaneous tissue are raised as a flap, protecting any obvious cutaneous nerve branches and reflected upward. Then, dissection of the underlying myofascia. This layer, including the deltopectoral muscle attachment, is raised as contiguous flaps and is preserved so that a twolayered closure can be achieved over the plate.

The fracture site is exposed by fully dissecting the proximal and distal fragments. Butterfly fragments are fixed with inter-fragmentary screw. In cases of simple fractures, depending on the obliquity of the fracture, it could be possible to place an inter-fragmentary screw across the fracture site which will improve the fracture fixation. The medial and lateral fragments of the fracture are held with reduction clamps to realign and maintain the reduction.

When the fracture reduced, the plate is applied and secured to the medial and lateral fragments. The plate should be bent to follow the contour of the clavicle. A drill-and-tap preparation is used to fix the plate to the clavicle, three screws are placed on either side of the fracture to achieve purchase through all six cortices of bone. Plates used in this study are $3.5 \mathrm{ml}$ reconstruction plates and anatomical S-plates.

Once the screws have been applied, the field is irrigated with normal saline. Standard closure of the wound is then performed in layers with use 
of no. 0 absorbable sutures for the myofascia and for the subcutaneous tissue, and no. 1 non-absorbable sutures for the skin. After the surgery, the arm is placed in a sling or shoulder immobilizer.

\section{Post-operative care:}

Patients were transferred to the ward where antibiotics, analgesics and anti-edema drugs were started. After recovery from anesthesia; injured limb was checked for neurovascular status. Blood soaked wound dressing changed. An anteroposterior and 45 degree cephalic tilt X-ray views of the clavicle were done for all patients. Patients were discharged after one day. A discharge sheet including follow-up duration and outpatient visits was given for the patient.

Patients were instructed to immobilize their arm in a sling, Continue on antibiotics, analgesics and anti-edema drugs for 10 days. Pendulum shoulder exercises are started during the first week.

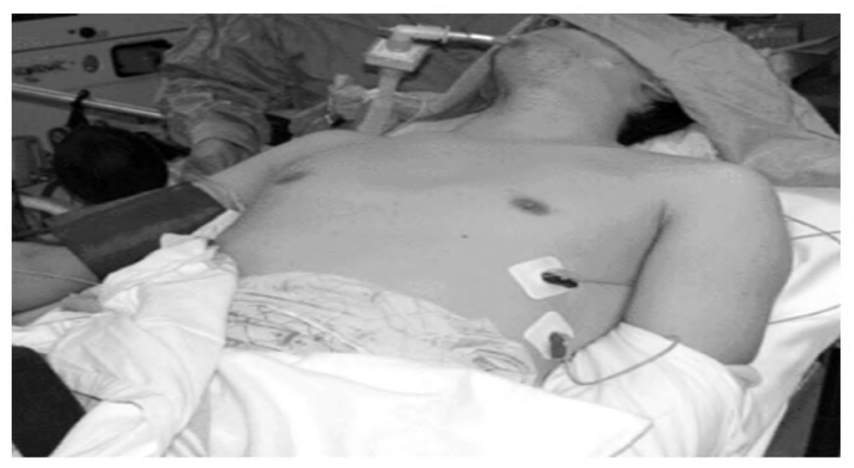

Fig. (1): The patient in the semi-sitting position, arm was draped at the side.
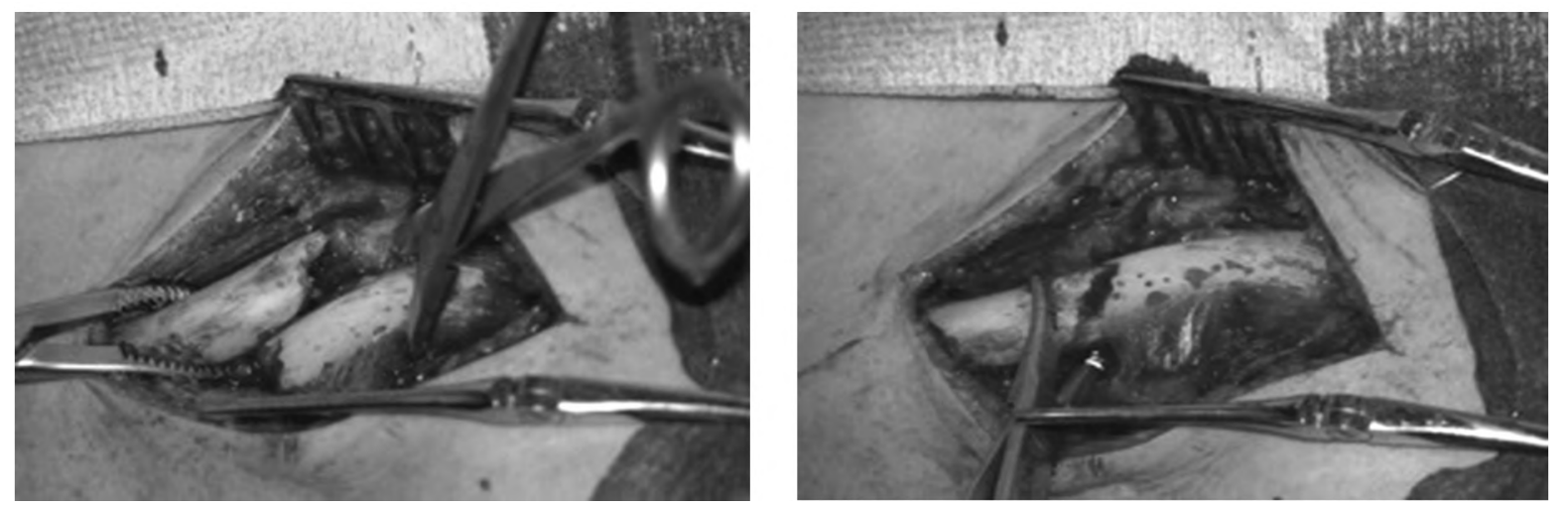

Fig. $(3,4)$ : Reduction is made and maintained by reduction clamps.

\section{Imaging:}

Follow-up X-rays were done every two weeks for the first two months (total $4 \mathrm{X}$-rays) and every month till evidence of strong union.

\section{Methods of patient assessment:}

Functional outcome was assessed using the Constant Shoulder Score (CSS) [33] adopted by the European Society for Shoulder and Elbow Surgery (ESSES). It consists of four variables that are used to assess each shoulder separately. The subjective variables are pain and activity of daily living (ADL) which give a total of 35 points. The objective variables are range of motion and strength that give a total of 65 points.

A difference between normal and abnormal side more than 30 is considered "poor", from $21-30$ is considered "fair", from 11-20 is considered "good" and less than 11 is considered "excellent" Constant Shoulder Score [33].

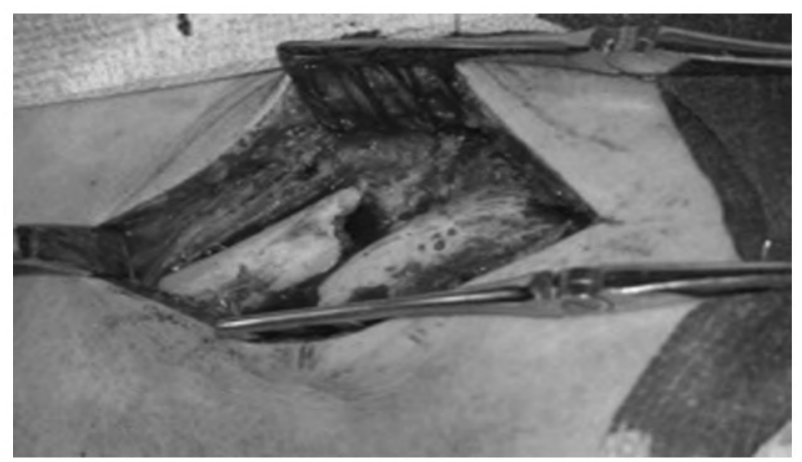

Fig. (2): The fracture site exposed. 